\title{
BRUNO TAUT: EL EDIFICIO DE LA FACULTAD DE ANKARA Y EL PALACIO KATSURA DE KIOTO. LA IDEA DE LA ARQUITECTURA EN EL CONCIERTO DE CULTURAS'
}

\author{
Manfred Speidel
}

\begin{abstract}
Los viajes de ida y vuelta que Bruno Taut y su esposa realizaron en su huida de la Alemania nazi hacia oriente le permitió adquirir una visión cosmopolita de la arquitectura que pudo plasmar durante su estancia en Japón en un voluminoso tratado, Architekturüberlegungen-Reflexiones sobre la arquitectura, al que luego sumaría Architekturlehre-Enseñar arquitectura.
\end{abstract}

Palabras clave: Bruno Taut, Japón, Turquía, Ankara, Alemania, viaje

Keywords: Bruno taut, Japan, Turkey, Ankara, Germany, travel

El arquitecto al emán B runo Taut (1880-1938) es muy conocido por sus grandes edificios residenciales en el B erlín de los años veinte, como la urbanización La Herradura. En 1932 fue a trabajar durante un año a M oscú, donde diseñó enormes hoteles que quedaron sin construir. Después marchó a Japón huyendo de la A lemania nazi y permaneció allí durante tres años y medio. U no de los dos proyectos arquitectónicos que pudo completar en ese país fue una suite de tres habitaciones, de las que una era un cuarto tradicional japonés, para una vivienda de fin de semana en A tami, al oeste de Tokio (Fig. 1).

B runo Taut y su esposa, Erica, dejaron Japón en barco el 15 de octubre de 1936. El viaje Ios Ilevó a Corea, a M ukden (hoy llamada Shenyang, en la región histórica de M anchuria) y a Pekín. Desde allí se dirigieron a Chitá (en la antigua Unión Soviética), donde tomaron el Transiberiano hacia M oscú para luego encaminarse hacia el sur, rumbo a O desa, hasta llegar a Estambul el 10 de noviembre.

De hecho, el itinerario que siguieron de Chitá a Estambul fue el mismo, en sentido contrario, que los había llevado desde Berlín a Japón en marzo de 1933 para escapar de la A lemania nazi. A causa de este inesperado viaje, Taut había visitado Zúrich, París y M arsella; y había navegado hasta Nápoles para recalar en A tenas antes de llegar a Odesa y coger el tren hacia M oscú. Exploró la arquitectura clásica de G recia y la otomana de Constantinopla y tomó conciencia de los problemas de la modernización y de la arquitectura moderna de Rusia, Turquía y Japón. Seguramente esto le permitió adquirir una visión cosmopolita de la arquitectura que pudo plasmar durante su estancia en Japón en un voluminoso tratado, Architekturüberlegungen [R eflexiones sobre la arquitectura], al que luego sumaría Architekturlehre [Enseñar arquitectura] y que fue traducido al turco como Mimari Bilgisi (Fig. 2) para convertirse en el manual de referencia de los estudiantes de la A cademia de Estambul que asistían a sus clases en 1937 y 1938. L amentablemente, no se imprimió hasta finales de 1938 y vio la luz tan solo dos semanas después del fallecimiento de Taut el 24 de diciembre, con tan solo cincuenta y ocho años.

Taut introdujo en este texto la perspectiva de una visión globalizadora en la que incluyó todas las obras más ilustres de la arquitectura mundial que había explorado durante aquellos años. Para él resultaba evidente que cada una de las grandes culturas había desempeñado una labor concreta cuando se analizaban bajo el prisma de la técnica, la estructura y la función.

Por ejemplo, en el libro considera que la arquitectura griega clásica alcanza la maestría técnica en el tratamiento de los materiales pétreos y en la ejecución del acabado de los detalles; también encuentra la misma maestría técnica y de refinamiento estético en las construcciones japonesas de madera y admira la vivienda japonesa en general por cómo

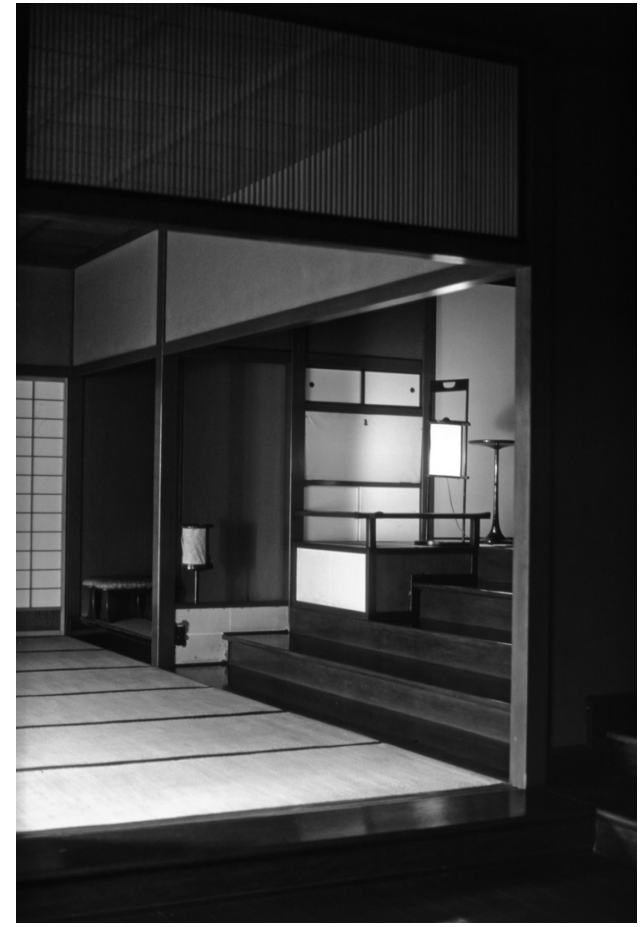

Fig. 1. Bruno Taut. Cuarto tradicional japonés en Villa Hyuga, Atami, 1936. Fotografía: Speidel.
1. Este artículo es una reelaboración de mi ensayo Bruno Taut. Wirken und Wirkung [Bruno Taut. Obra e influencia], en Atatürk için düşünmek. Für Atatürk gedacht. Thinking for Atatürk. Estambul: Milli Reasurans T.A.S., 1997, pp. 54-61. 


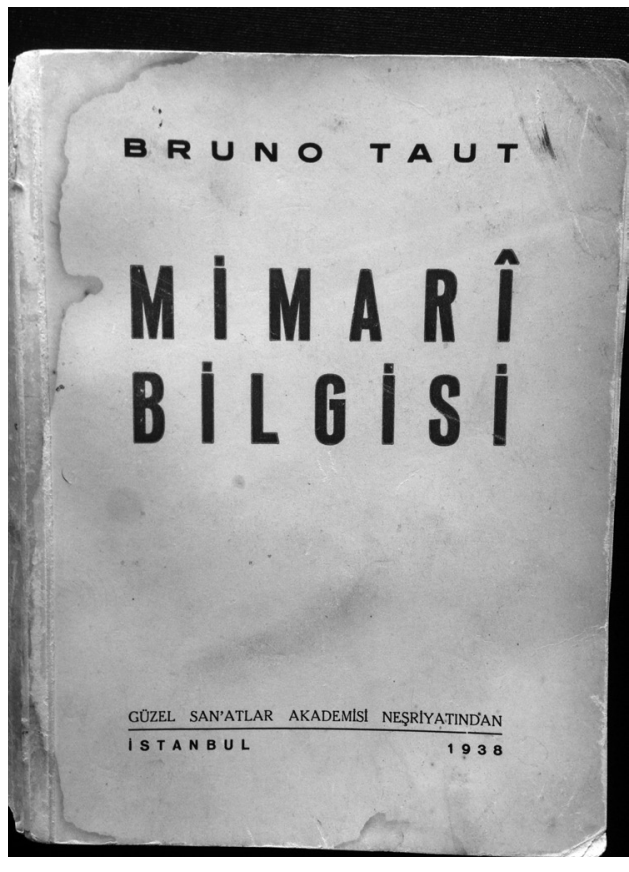

2

Fig. 2. Bruno Taut. Mimari Bilgisi [Enseñar arquitectura] 1938, Estambul.

Fig. 3. Palacio Katsura, Kioto, Shoin Antiguo, s. xvII. Extraído de YOSHIDA, Tetsuro, Das japanische Wohnhaus. 1935, Berlín: Wasmuth, p. 35

Fig. 4. Bruno Taut. Facultad de Lenguas, Historia y Geografía, Ankara, 1937-1939. Vista global. Fotografía: Speidel.

Fig. 5. Bruno Taut. Colegio de Trabzon, 1937-1939. Fotografía: Speidel.

Fig. 6. Bruno Taut y Gonkuro Kume. Villa Okura, Tokio, 1936. Fig. 7. Bruno Taut. Edificio de la facultad, Ankara. Cuerpo central y entrada. Fotografía: Speidel.

Fig. 8. Facultad de Lenguas, Historia y Geografía. Perspectiva. Con fecha de 20.11.1937. Extraído de "Projects of the Office for Realisation at the Academy of Fine Arts". Álbum documental. Colección Speidel.

2. El análisis de Katsura permitió a Taut profundizar en su idea de relatividad ("arquitectura de relaciones"), así como en la de "elasticidad" de las funciones y en su concepción final de la arquitectura como el arte de la "proporción".

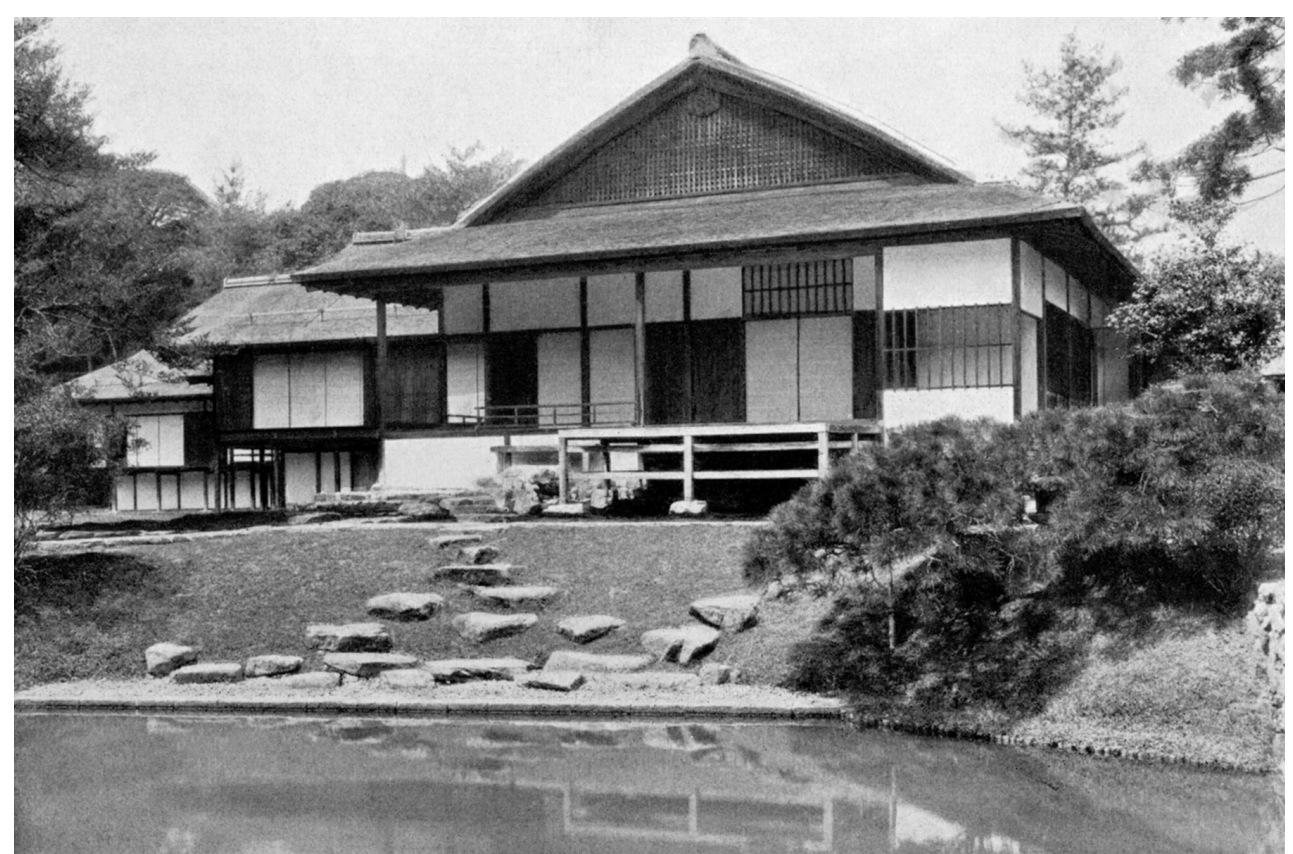

3

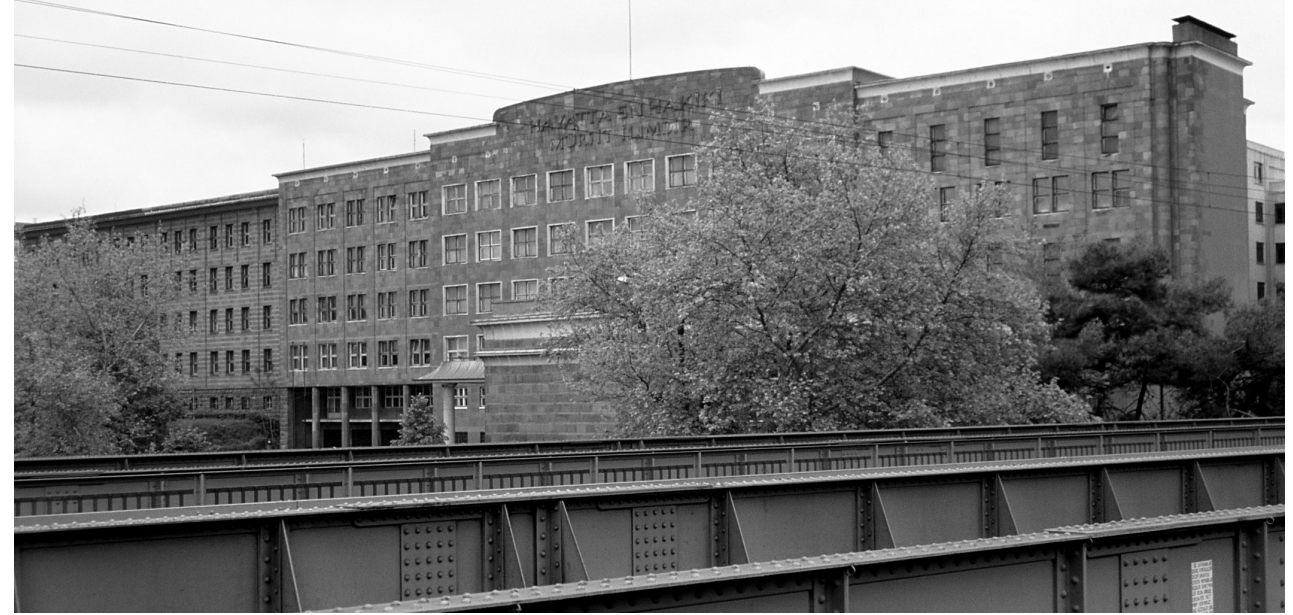

4

se controlan las altas temperaturas y la humedad de su clima veraniego mediante muros correderos y la posibilidad de una apertura total. La arquitectura gótica había destacado en cuestiones estructurales, al igual que la turca, con obras maestras que presentaban enormes estructuras, bóvedas y cúpulas de piedra. La variedad y la jerarquía de los aspectos funcionales podían analizarse mejor en los conjuntos de edificaciones y jardines del Palacio K atsura de $\mathrm{K}$ ioto² (Fig. 3). Taut asignó a cada cultura una importante contribución a la arquitectura mundial respecto a otras culturas y reemplazó las interpretaciones nacionalistas de superioridad cultural por la idea de que cada cultura efectúa una aportación única a lo que hoy denominamos "patrimonio de la humanidad". M e gustaría Ilamarlo "la creación de un concierto de culturas".

Cuando Taut llegó a Estambul el 10 de noviembre de 1936 ya había negociado un contrato y empezó a trabajar al día siguiente en la Academia de Estambul y en el Área de A rquitectura del M inisterio de Cultura. Tan solo tres semanas más tarde, el 1 de diciembre, le encargaron el diseño de la Facultad de Lenguas, Historia y Geografía de la U niversidad de A nkara. A parte de la complejidad programática, se trataba del edificio más importante y representativo de la "N ueva Turquía" de Kemal A tatürk (Fig. 4). 
Por otra parte, tuvo que construir varios colegios en Ankara y en algunas provincias. Taut tenía experiencia en edificios escolares de sus años en A lemania, como el instituto de Senftenberg, al sur de B erlín. De hecho, pudo aplicar una tipología similar cuando diseñó el colegio de Trabzon, donde adecuó a un clima más cálido los grandes huecos de la fachada sur añadiendo a las ventanas unos parasoles cuya cara superior reflejaba la luz hacia el techo de las aulas (Fig. 5), parasoles que ya había utilizado en la Villa Okura de Tokio en 1935 para regular el calor y la humedad típicos de aquella región (Fig. 6). Taut estaba orgulloso de haber quebrantado la caja blanca moderna y formalista de estilo cubista-Le Corbusier que había propuesto su colaborador, Kume. En realidad, este había sido su único proyecto arquitectónico en J apón aparte del diseño de interiores. Paralelamente, había producido unos doscientos cincuenta objetos de uso cotidiano y mobiliario de la mano de artesanos tradicionales en talleres locales.

Lo que no tenía Taut era ninguna solución ya experimentada y a mano para el edificio universitario de A nkara. Tras cuatro años de abstinencia en J apón en los que no había podido diseñar edificios de entidad, Taut estaba entusiasmado por profundizar en una concepción de la arquitectura en el contexto de esa cultura para la que ahora trabajaba y que, por supuesto, era muy diferente a la de A lemania o Japón, pero quiso incorporar sus experiencias en aquellos países.

Si el libro Mimari Bilgisi podría considerarse el legado teórico de Taut, o más explícitamente la integración de la arquitectura mundial en una teoría de la relatividad, la Facultad de L iteratura de la U niversidad de A nkara iba a reflejar sus convicciones sobre el lugar que debe ocupar un edificio público importante en el contexto social y cultural para poder llegar a considerarse una contribución "al mundo en su conjunto", como exigía a la buena arquitectura.

El 6 de noviembre de 1937, Taut escribió a su colega Kurata, que vivía en Tokio: "En este momento, lo que me preocupa es verificar mi actual concepción de la arquitectura". A un teniendo presente todo esto no resulta fácil valorar el edificio de la Facultad de Lenguas, Historia y Geografía (Figs. 4, 7-12).

Con unos ciento cuarenta metros de longitud a lo largo del bulevar A tatürk y seis plantas de altura, su reducida profundidad y su mínima gradación confieren al edificio la apariencia de un del gado muro urbano que limita el nuevo campus universitario. El volumen principal está compuesto por un cuerpo central ligeramente adelantado cuya longitud duplica la de cada uno de los dos laterales simétricos, más un volumen adicional a la izquierda en forma de $L$ que define una esquina del campus.

El cuerpo central está a su vez compuesto por tres elementos (Fig. 7). Sobre la disposición regular de ventanas cuadradas de la lisa fachada se el eva un hastial ancho y ligeramente curvo que interrumpe la potente cornisa de la cubierta plana. El hastial se asemeja a un escudo, con el lema y símbolo del impulso cultural de A tatürk que, traducido al español, dice: "En la vida, el auténtico líder es el conocimiento" (el diseño de la tipografía es de Taut).

La prominente fachada no parece muy estable sobre los dos muros curvos de la planta baja. Como si formaran una gran boca, atraviesan esta fachada principal y conducen a los estudiantes hasta el interior del edificio y hacia la colina del campus que se encuentra al otro lado. Son una especie de portalada de acceso al campus.

Desde un punto de vista compositivo, el riguroso al zado queda de este modo brutal mente perforado por el porche de entrada, cuya cubierta se extiende para cubrir también la antesala del auditorio - que sobresale del plano de fachada- y se prolonga componiendo los volúmenes más bajos del auditorio y dependencias secundarias hacia el bulevar (Fig. 8).

Este porche con una única y voluminosa columna en la esquina abierta acentúa un movimiento diagonal desde el espacio público hacia el pasadizo y el vestíbulo interior. A simismo, los muros del auditorio se curvan para suavizar el recorrido hacia la antesala (Fig. 9). Este movimiento continúa en la escalera hacia la galería del auditorio, que arranca con un pasamanos helicoidal, un remolino que parece invitar al baile (Fig. 10). En el

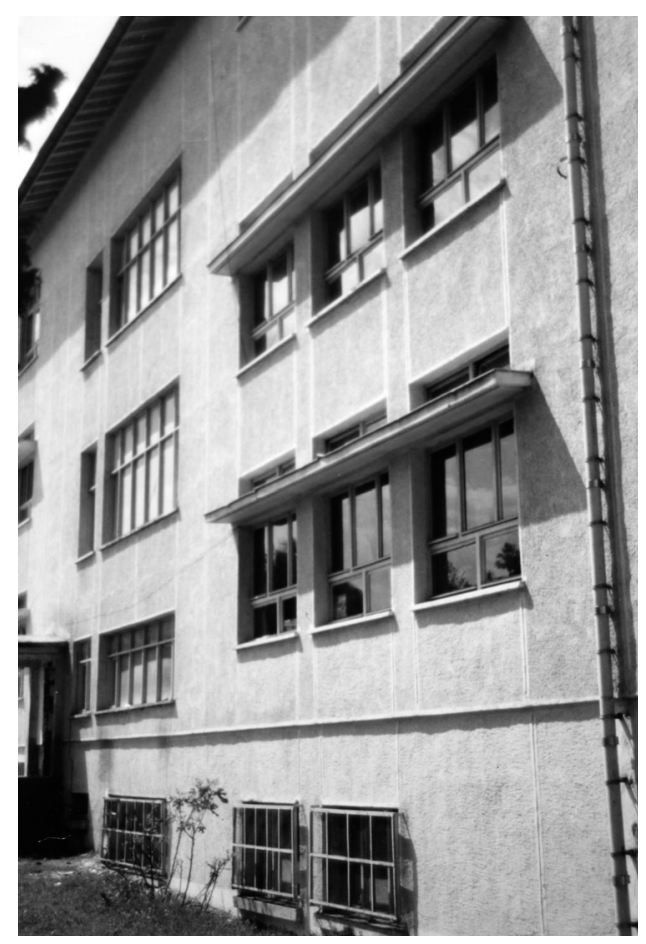

5

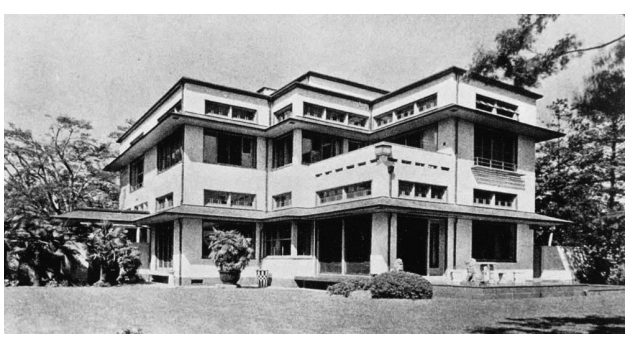

6

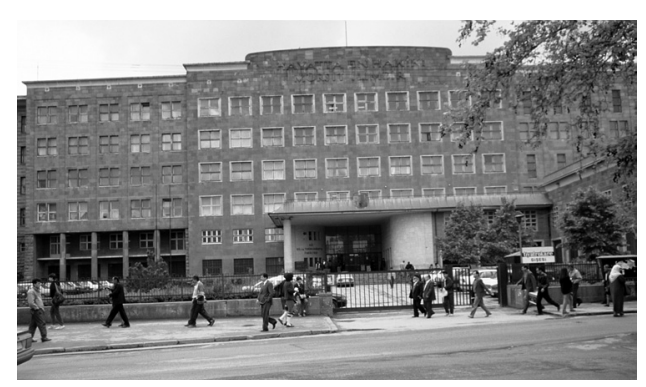

7

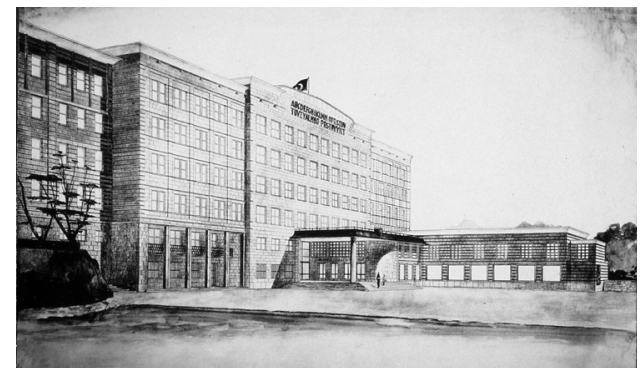

8 


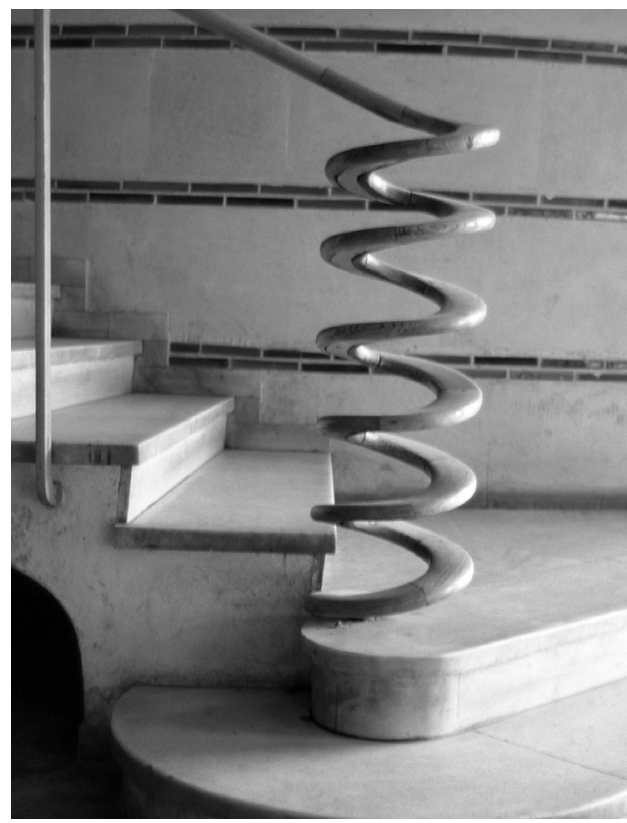

10

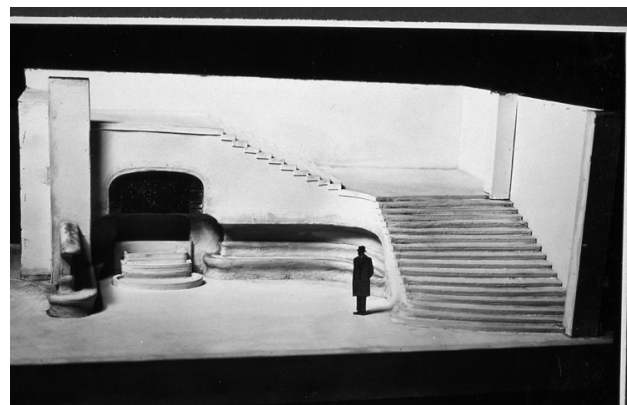

11

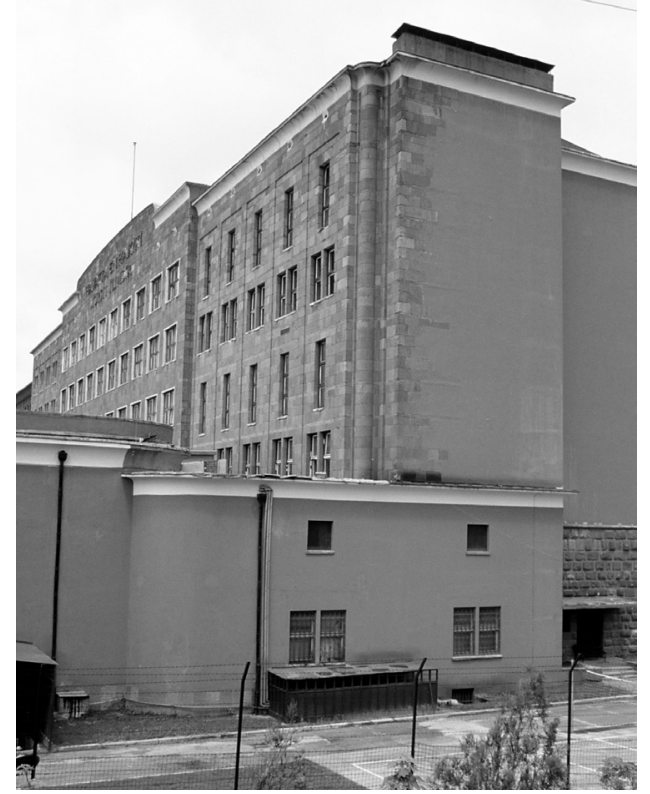

12

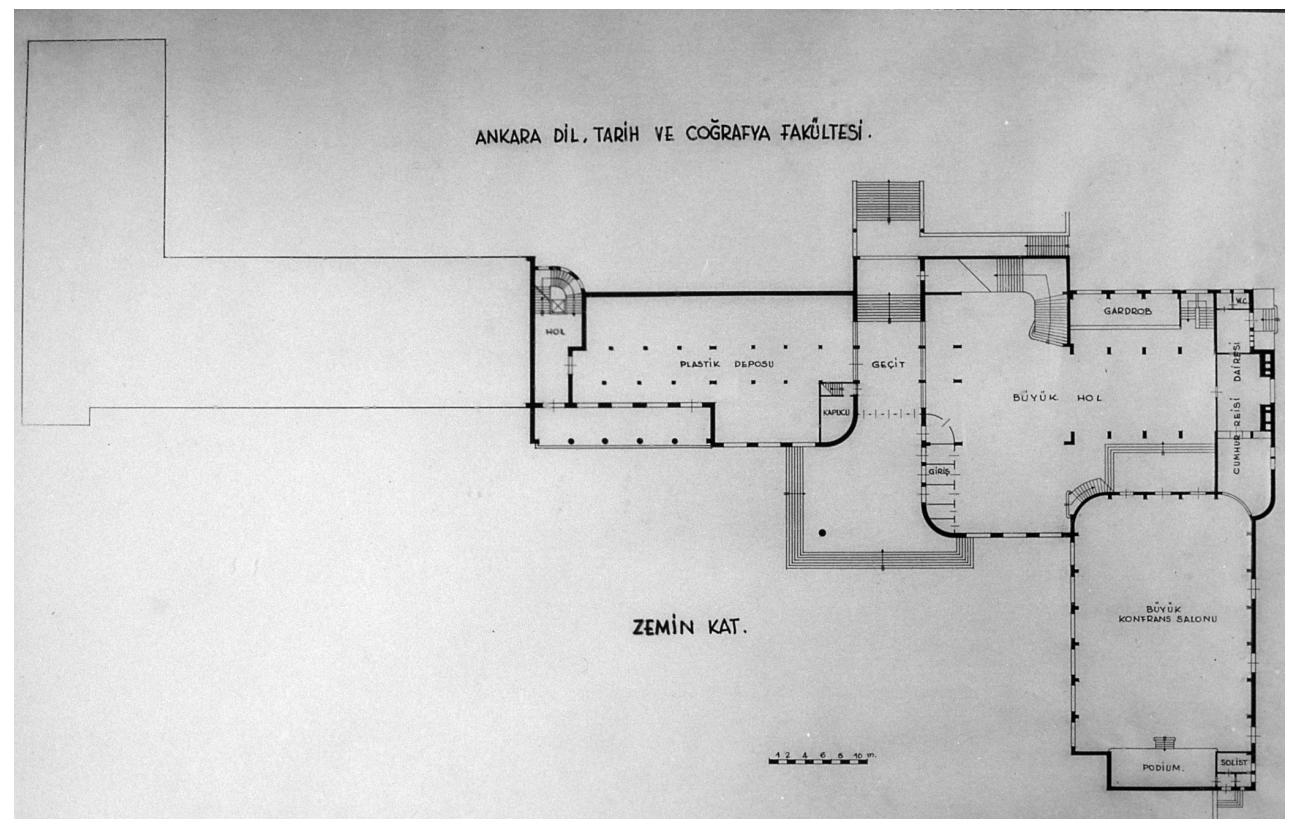

9

Iado opuesto del vestíbulo, los primeros peldaños de la escalera principal hacia las aulas superiores se giran levemente hacia la dirección diagonal de la entrada. Taut quería incluir una escultura "orgánica" en forma de cueva bajo este primer tramo, que sirviera de base a las atrevidas líneas ascendentes y que incorporara una fuente con un banco curvo para descansar en medio del flujo constante de personas (Fig. 11). Sin embargo, esto último no llegó a construirse.

En el exterior, puede resultar extraño el final tan abrupto del extremo derecho del edificio (Fig. 12). Da la sensación de que se hubiera previsto una ampliación posterior, ya que solo media columna parece indicar que esta esquina es un el emento de transición. ¿0 quizá aTaut le gustaba este carácter singular? No lo sabemos.

El interés de Taut por enfatizar recorridos múltiples y fluidos dentro y alrededor del edificio, en la calle y planta baja, se contradice desde un punto de vista compositivo con la estricta composición de las formas exteriores de la parte central del edificio que, a su vez, presenta ciertas irregularidades: las columnas sin decorar de la izquierda y las ventanas de la planta superior del auditorio. Podemos preguntarnos en qué se inspiró Taut para esta inusual composición. ¿Quizá se debiera a la complejidad del programa, a la propia vida interna del campus y a la obligación añadida de permitir el acceso público a las conferencias, tal y como exigía el lema de A tatürk para educar a las masas en la Turquía moderna?

Desde mi punto de vista, antes de ir a J apón Taut empleaba mecanismos mucho más coherentes para combinar una fachada regular con un elemento irregular, como en la Sede de la A sociación A lemana de Transportes y Comunicaciones de Berlín (1927-1932). Sobre la entrada principal añadió a las dependencias del equipo directivo una especie de mirador que no interrumpía la composición horizontal de la estructura. El diseño para el hotel Intourist de M oscú (1932) exhibía una composición más equilibrada de enormes cuerpos verticales y un volumen horizontal que completaba el edificio.

Quizá sorprenda mi propuesta de que la espontánea composición de A nkara es el resultado del análisis de Taut sobre el Palacio de Retiro Imperial Katsura de Kioto, construido durante el siglo xVII (Figs. 3, 13-17).

Taut visitó el palacio durante su segundo día en Japón, el 4 de mayo de 1933. Quedó sorprendido por la rectitud de un diseño aparentemente simple y por la combinación de elementos simétricos más rigurosos con una composición global más libre de los edificios y su relación con el amplio jardín. 


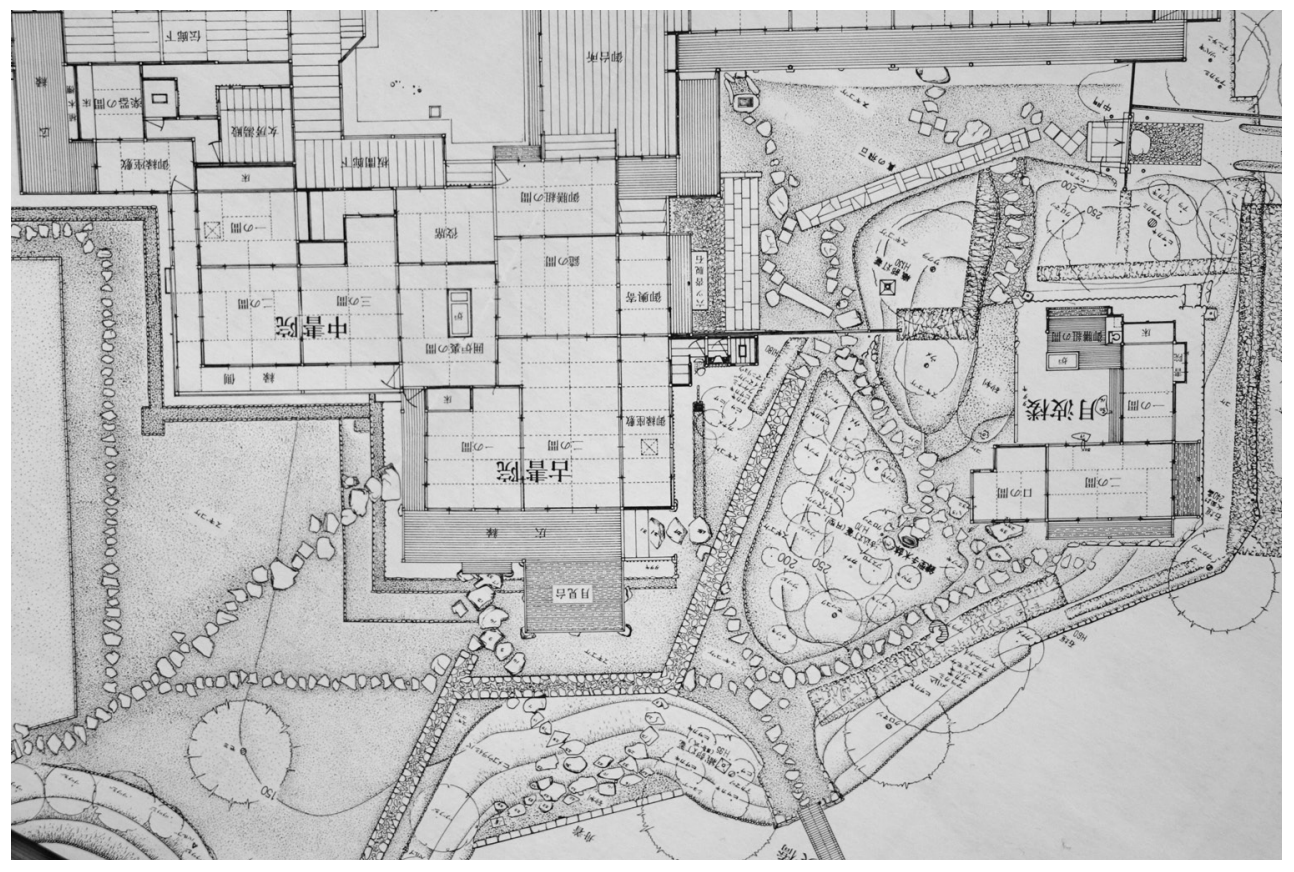

14

El Palacio K atsura está formado por tres cuerpos conectados, conocidos como pabellones de estilo shoin. El principal se denomina Shoin A ntiguo (1617), y era el edificio en que recibían a sus invitados nobles y en el que daban comienzo las fiestas que continuaban en el jardín, donde navegaban por el lago mientras leían poemas (Fig. 3). Tiene una cubierta a cuatro aguas con un gran hastial frente al jardín y al lago con su isla, de exquisito diseño. El prominente hastial del Shoin A ntiguo, ligeramente curvo y convexo, está adornado con el blasón familiar, y es un símbolo de su dignidad y carácter representativo al que se subordinan los pabellones contiguos de la izquierda, que sucesivamente dan un paso atrás y, al estar girados noventa grados, muestran el al ero para crear una secuencia libre de todas las partes. Estos pabellones laterales son el Shoin M edio y el denominado "Nuevo Palacio".

Bajo el hastial simétrico y los aleros de la cubierta inclinada, las habitaciones se disponen asimétricamente, lo que se manifiesta en el exterior por la secuencia irregular de los pilares que conforman el porche y soportan la cubierta. Podría definirse como una simetría blanda o mitigada, ya que se limita a la cubierta y desaparece bajo ella. En cualquier caso, la asimetría de los pilares resulta bastante natural.

Fuera del porche y a un lado del eje se ha colocado un mirador para contemplar la luna, una estructura ligera de madera desde la que, en otoño, puede disfrutarse del reflejo de la luna llena en el estanque (Fig. 13). En el extremo derecho del porche, un pequeño muro esconde unos peldaños de piedra que bajan al jardín, por lo que toda la composición del alzado confiere fluidez espacial al movimiento por este espacio cubierto (Fig. 14). J unto al porche, un enlosado de superficie rugosa da acceso al jardín a través de senderos radiales, de piedras irregulares aisladas, que conducen directamente al estanque 0 al jardín. Los cruces de estos senderos entre sí y con el camino recto pavimentado, que pasa tangencialmente desde el patio de entrada hasta la isla de una de las casas de té, configuran una red funcional de recorridos diagonales de especial utilidad en un suelo embarrado tras los días de lluvia.

Taut interpreta la irregularidad con la que se entrecruzan los senderos frente al Shoin A ntiguo como indicio de una sociedad libre e ilustrada (Fig. 15). En un dibujo en el que analiza el edificio en 1934, anota: “¿POR QUE ninguna línea del pabellón se prolonga hacia el jardín? - Porque cada uno de los elementos: el pabellón, el agua, el embarcadero, el árbol y la piedra vive su propia vida. Ú nicamente procuran mantener una buena relación, como en una buena sociedad". En otro texto sobre K atsura, concluye: "Podemos imaginar que, si las formas presentan tal naturalidad, la manera de actuar y de relacionarse de las personas estaría igual mente caracterizada por la rutina y la naturalidad" 3 .

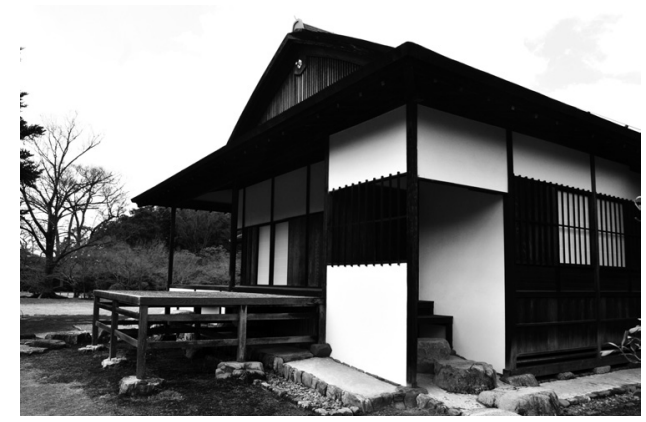

13

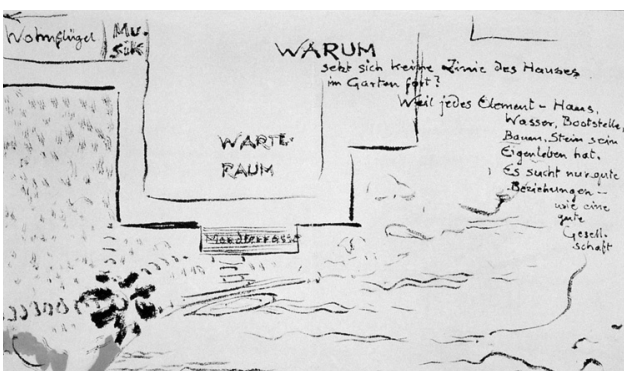

15

Fig. 9. Edificio de la facultad, plano de planta baja del vestíbulo de entrada y el auditorio. Álbum documental. Colección Speidel.

Fig. 10. Escalera hacia la galería del auditorio. Fotografía: Speidel.

Fig. 11. Maqueta de la escalera principal, fuente y banco. Álbum documental. Colección Speidel.

Fig. 12. Edificio de la facultad, detalle de la esquina. Fotografía: Speidel.

Fig. 13. Palacio Katsura. Shoin Antiguo. Fotografía: F. Morero. Fig. 14. Palacio Katsura. Planta, cartel anunciador para un libro sobre jardines japoneses.

Fig. 15. Bruno Taut. Extraído de Álbum de Katsura, 1934. Impresión facsímil. 1981, Tokio: Iwanami Shoten.

3. TAUT Bruno, NIPPON mit europäischen Augen gesehen [Japón visto con ojos europeos], 1934, SPEIDEL, Manfred (ed.). 2009, Berlín: Gebr. Mann, p. 28. 


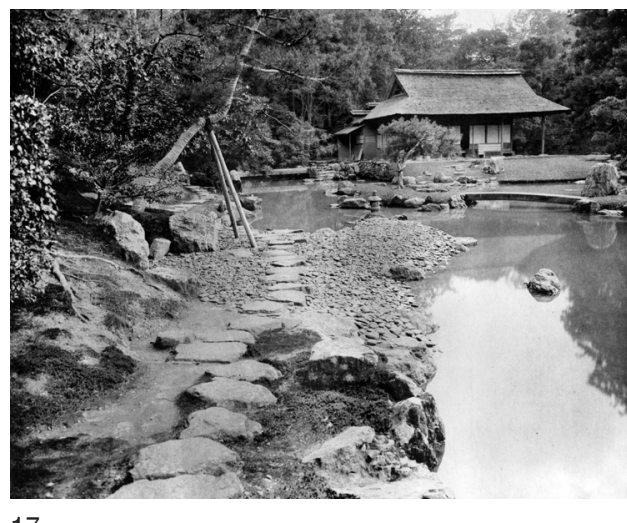

17

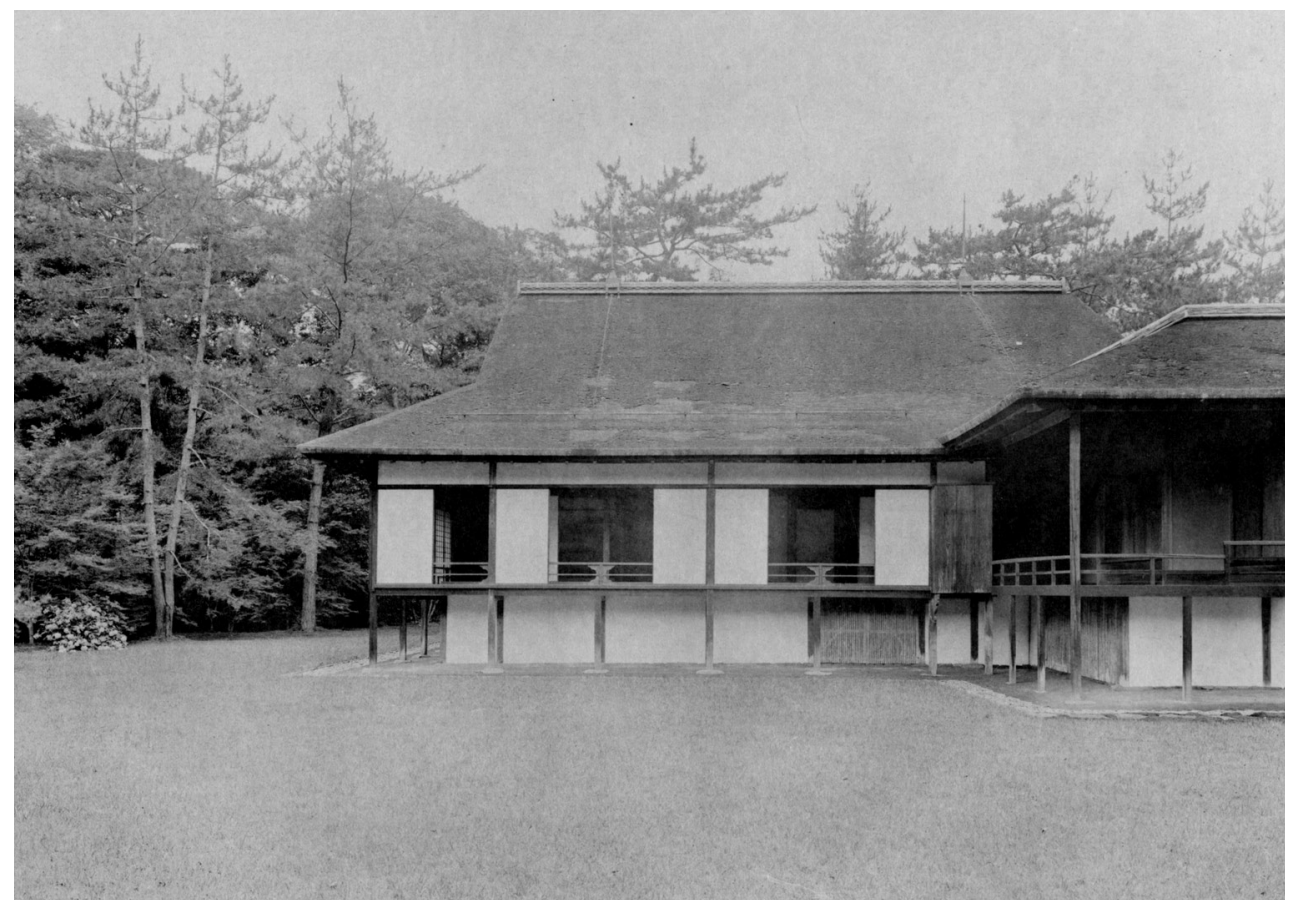

16

Hay que señalar que esta naturalidad se organiza alrededor del edificio al que Taut reconoce una función representativa de la sociedad, simbolizada por la cubierta simétrica dominante, lo que en cierto modo es contradictorio, ya que albergaba a los miembros de una sociedad feudal (es decir, ordenada jerárquicamente) y la tipología del pabellón shoin pone de manifiesto esta jerarquía feudal. Por otra parte, los dos cuerpos laterales para la vida diaria presentan un exterior bastante austero y no tienen vistas a ningún jardín expresamente diseñado, sino a un sencillo césped al que no tienen acceso directo. Se construyen sobre pilotis debido a las inundaciones periódicas (Fig. 16).

La similitud más Ilamativa entre K atsura y el edificio universitario de A nkara es la combinación de la función representativa que domina la parte superior de la fachada con la naturalidad de los recorridos que se dejan guiar por elementos arquitectónicos en la planta baja. L a simetría de la fachada de la facultad puede considerarse -igual mente- blanda o mitigada, ya que se matiza en el cambio de la forma de las ventanas: en el cuerpo central se mantienen en el mismo plano, recercadas con una fina línea, mientras que en los cuerpos laterales se rehúnden levemente (Fig. 7).

Para elevar la fachada mediante un escudo con inscripciones sobre la entrada principal, Taut pudo haberse inspirado en las portadas de las mezquitas turcas. Sin embargo, el dominio del hastial curvo recuerda mucho al de Katsura, donde el blasón familiar ocupa una posición simbólica similar a las palabras de A tatürk. Paral elamente, la interpretación de Taut sobre K atsura nos permite apreciar que la distribución no convencional de la planta baja de la facultad reivindica una sociedad de individuos libres e ilustrados. Si Taut tuvo la valentía de combinar la monumentalidad del rigor de la fachada con un esquema irregular para la vida diaria y los recorridos de la planta baja, fue gracias - estoy convencido de ello- a la meticul osa observación del Palacio K atsura de Tokio (Figs. 13 y 8).

L as amplias ventanas cuadradas dispuestas ordenadamente en la parte central de la fachada de la Facultad de Lenguas, que configuran una retícula neutra, ocupan un lugar formalmente intermedio entre el hastial simétrico y la composición de distintos volúmenes frente a la entrada, al igual que sucede, en mi opinión, con la retícula y el módulo de las puertas correderas en el porche de Katsura, ya que condicionan los pilares que soportan la cubierta.

Este breve análisis puede no constituir una prueba irrefutable, pero encuentro interesante y obvio que, cuando Taut transfirió estos el ementos de una cultura a otra, no tenía en mente 


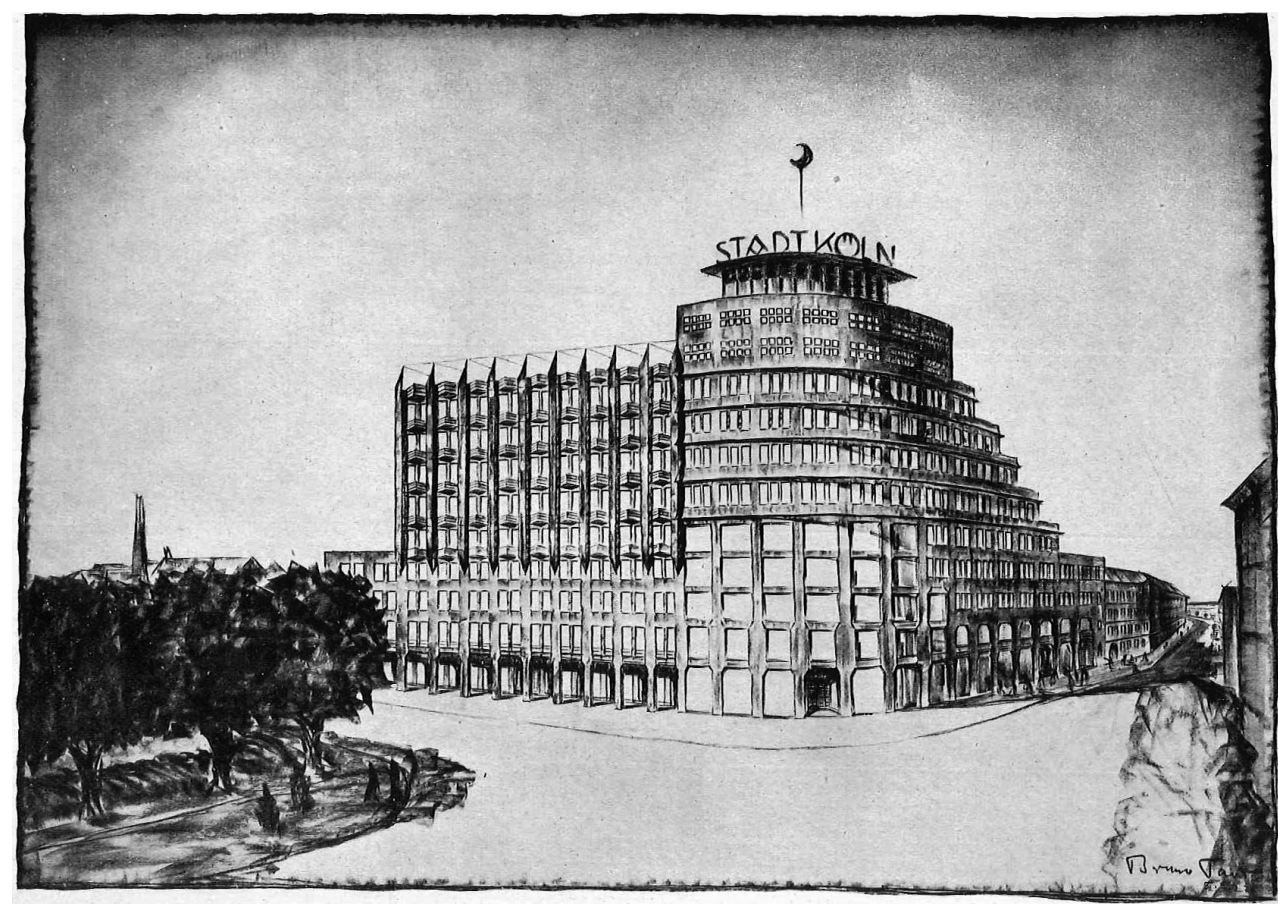

18

un estilo, sino una concepción de su papel en la composición arquitectónica. Demostró una gran habilidad para extraer conceptos abstractos de la realidad arquitectónica que había explorado y cuyo descubrimiento le había emocionado tanto.

Creo que gracias al estudio de la arquitectura de distintas culturas pudo establecer cuatro líneas directrices: La primera es que apreciaba una heterogeneidad en la composición que de hecho no agradaba ni a modernos ni a tradicionalistas. Desde que se convirtiera en el arquitecto de la Sociedad A lemana para la Ciudad J ardín en 1913, y especialmente durante sus años como consejero de urbanismo de la ciudad de Magdeburgo de 1921 a 1924, Taut había seguido un camino que no insistía en principios formales de estilo, sino que buscaba para cada proyecto y para cada una de sus partes una forma "esencial" o característica, es decir, funcional. Taut defendía su visión de "principios sin principio" en sus conferencias de 1923, cuando tenía que explicar al público sus diseños para M agdeburgo, que solían ser dispares y faltos de homogeneidad. Ponía como ejemplo su proyecto no construido, Stadt Köln, un hotel y edificio de oficinas en esa misma ciudad; una especie de amal gama de partes de edificios de diferentes diseños que al bergaban oficinas, habitaciones de hotel, apartamentos y tiendas, unificados en una esquina redondeada gracias a una fina cubierta circular que se adelantaba sobre la fachada y a unas grandes letras en lo al to (Fig. 18).

Cabe señalar que Taut citaba unas frases de El libro del té, escrito en 1906 por Okakura Kakuzo y publicado en versión al emana en 1919. O kakura escribe4:

\footnotetext{
La naturaleza dinámica de su filosofía (Taut añade: "taoísta y zen") consideraba más importante el proceso por el cual se buscaba la perfección que la perfección misma. La verdadera belleza podía ser descubierta solo por aquel que completara mentalmente lo incompleto. El vigor de la vida y el arte consistía en sus posibilidades de crecimiento. [ ]

Se consideraba la uniformidad en el diseño como algo fatal para la frescura imaginativa.
}

La importancia del proceso y la antiuniformidad fueron las dos ideas del ensayo de Okakura que Taut extrajo y llevó a la práctica. La segunda línea puede ser su concepción de una jerarquía de los edificios de acuerdo con sus funciones y su estatus en la sociedad y en la comunidad urbana, su voluntad de definir el lugar adecuado que debe ocupar el proyecto en el contexto de esa sociedad. Es una idea que Taut ya había formulado en 1920 y que creyó haber visto materializada en el Palacio K atsura de Japón. En K atsura identifica tres funciones: "Toda la planta [... ] obedecía de manera flexible en todas sus partes la finalidad que debía cumplir cada una de esas partes y también el conjunto de todas ellas, ya fuera esa
Fig. 18. Bruno Taut. Diseño para hotel y edificio de oficinas Stadt Köln en Magdeburgo. Extraído de Frühlicht, núm. 4 (1922), p. 121
4. Las frases se extraen del capítulo 4: "The Tearoom". KAKUZO Okakura, The Book of Tea, MURAOKA, Hiroshi (ed.). 1939, Tokio: Kenkyusha, p. 62. Traducido al español por María Teresa Solá como "El recinto del té" en El libro del té. 1992, Buenos Aires: Leviatán. Taut reprodujo la cita en sus conferencias de 1923: Vom gegenwärtigen Geist der Architektur [Sobre el espíritu del presente en la arquitectura] en SPEIDEL, Manfred, Bruno Taut. Ex Oriente Lux. 2007, Berlín: Gebr. Mann, p. 160-167. 


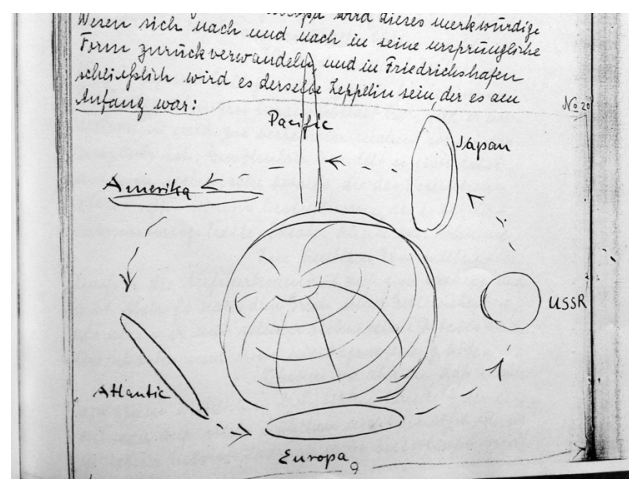

19

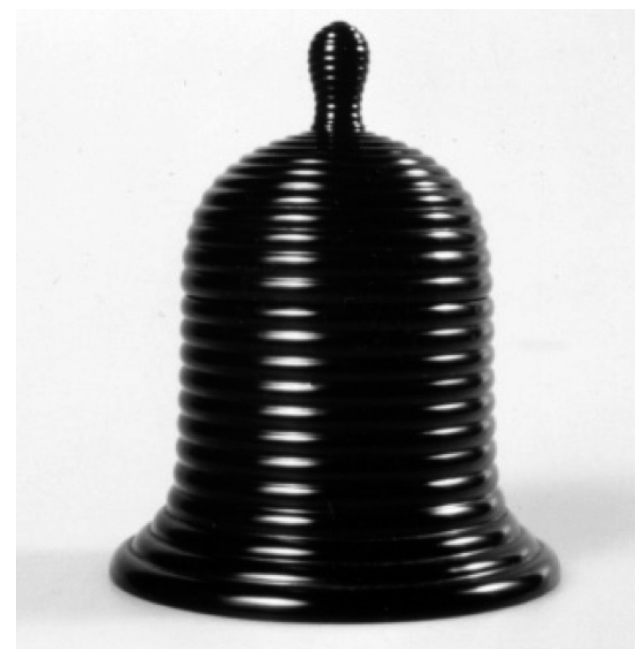

20

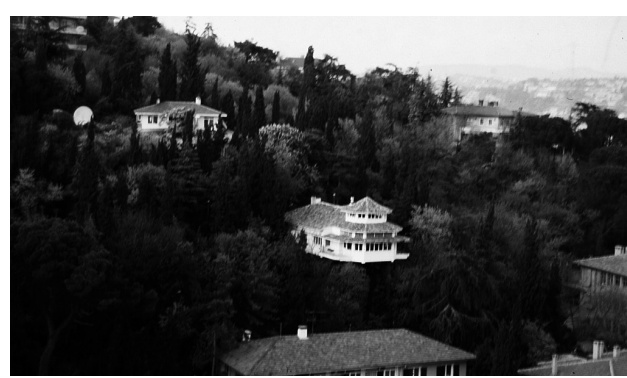

21

Fig. 19. Dibujo extraído de Mimari Bilgisi [Enseñar arquitectura], 1937. Manuscrito, Tokio: Iwanami Shoten.

Fig. 20. Bruno Taut. Pitillera de madera y laca, 1934. Museo de Historia de la Prefectura de Gunma, Takasaki.

Fig. 21. Bruno Taut. Su vivienda en Ortakoy, Estambul, 1938 Fotografía: Speidel. función la de la utilidad cotidiana normal, la de una representación digna o también la de una el evada espiritual idad filosófica (en la casa de té Shokintei con sus exquisitas vistas al jardín. M. S.). Y el milagro era que esas tres finalidades formaban una unidad tan perfecta que no se percibían límites entre ellas" 5 (Figs. 16, 3, 17). Taut estaba convencido de que estas tres funciones escalonadas: el uso cotidiano, la representación y la más alta espiritualidad artística eran necesarias para una sociedad cultivada. De hecho, en 1920 enumeró más de diez grados desde la sencilla cabaña en el peldaño inferior hasta la arquitectura como puro arte en el más elevado, cuya única función es ser bella. En K atsura, la casa de té y la ceremonia del té al canzaban la más alta posición.

La tercera línea directriz de la concepción arquitectónica de Taut sería la igualdad entre las diferentes culturas, una idea que desarrolló en su libro Architekturlehre. Una variante de ella es que las diferencias entre las soluciones arquitectónicas para una misma función, como las granjas, podrían derivar de sus condicionantes geográficos y climáticos. Taut dibuja un zepelín que vuela por todo el planeta mientras adapta su forma oblonga inicial hasta convertirse en circular y su dirección va pasando de horizontal a vertical. E ra el manifiesto de Taut en contra de cualquier tipo de "estilo internacional" uniforme (Fig. 19).

La cuarta línea podría identificarse con la cuidadosa utilización de el ementos arquitectónicos de tradición local para que no se conviertan en recursos románticos o de estilo.

Recién Ilegado a Turquía y sin hablar la lengua, el conocimiento de Taut sobre la historia y cultura turcas no podía ser tan profundo como el que tenía de Japón, sino que era bastante superficial en cierto sentido.

En Ankara, Taut incorporó algunos elementos de la tradición nacional. La piedra de la fachada provenía de una cantera local. La fina sección de los machones de las ventanas para modificar su forma cuadrada, así como la albañilería "turcobizantina", con hiladas de ladrillo que se alternan con piedra local en la prolongación del ala izquierda (la parte no representativa) hacen referencia a la arquitectura tradicional turca. L a fachada lateral finaliza con una columna decorada (un motivo turco), pero en la esquina se convierte bruscamente en un sencillo muro enfoscado, un detalle tradicional al emán que echa por tierra cualquier pretensión romántica 0 monumental y nos descubre que la sillería de piedra es una fina máscara (Fig. 12). Esto significaría que la tradicional fachada de estilo turco sería simplemente un bonito y el egante vestido, ¡un auténtico tabú para el movimiento moderno! Sin embargo, posteriormente se ha aceptado que el edificio de Taut inició el segundo estilo nacional de Turquía.

Tokugen M ihara, ayudante de Taut en J apón, señaló que, cuando en Japón diseñaba objetos de uso cotidiano como la pitillera de madera y laca japonesa que recordaba una cúpula oriental, Taut echaba mano de un imaginario íntimo compuesto por elementos de la cultura oriental y occidental (Fig. 20). M ihara estaba convencido de que "el hogar espiritual y el gran amor de Taut no fue J apón, sino Oriente Próximo, por el que sentía una verdadera fascinación. Japón llegó a su mente más tarde".

Los japoneses piensan que la vivienda que se construyó Taut en 1938 en Ortakoy es de estilo turco, y los turcos, que es japonesa. Taut incorporó nuevamente el parasol en los enormes cerramientos acristalados, pero empleó tejas turcas en la cubierta (Fig. 21).

M ihara concluía: "La vivienda se emplaza en la orilla europea del B ósforo. Parece suspirar por A sia... desde Europa"
5. TAUT, Bruno, Das japanische Haus und sein Leben, 1937, traducido al español por Dolores Ábalos como La casa y la vida japonesas, 2007, Barcelona: Fundación Caja de Arquitectos, p. 283.

6. MIHARA, Tokugen, Kunstgewerbe, nota 1, p. 142.
Manfred Speidel. Profesor de Arquitectura en la Universidad de Aachen y es un experto en arquitectura alemana y japonesa. Ha participado en numerosas conferencias sobre ambos temas y sobre la arquitectura de Bruno Taut en muchas instituciones de todo el mundo. Además, organiza en su universidad conferencias todas las semanas bajo el título "Charlas de arquitectura para los lunes por la noche". 